\title{
Quantum Chaos in a Yang-Mills-Higgs System
}

\author{
Luca Salasnich 円 \\ Dipartimento di Matematica Pura ed Applicata, \\ Università di Padova, Via Belzoni 7, I 35131 Padova, Italy \\ and \\ Istituto Nazionale di Fisica Nucleare, Sezione di Padova, \\ Via Marzolo 8, I 35131 Padova, Italy
}

\begin{abstract}
We study the energy fluctuations of a spatially homogeneous SU(2) YangMills-Higgs system. In particular, we analyze the nearest-neighbour spacing distribution which shows a Wigner-Poisson transition by increasing the value of the Higgs field in the vacuum. This transition is a clear quantum signature of the classical chaos-order transition of the system.
\end{abstract}

To be published in Modern Physics Letters A

\footnotetext{
${ }^{1} \mathrm{E}-$ Mail: salasnich@padova.infn.it
} 


\section{Introduction}

In the last years there has been much interest in classical chaos in field theories. It is now well known that the spatially uniform limits of scalar electrodynamics and Yang-Mills theory exhibit classical chaotic motion ${ }^{1)-8)}$. On the other hand, in field theories, less attention has been paid to quantum chaos, i.e. the study of properties of quantum systems which are classically chaotic $^{9)}$.

The energy fluctuation properties of systems with underlying classical chaotic behaviour and time-reversal symmetry agree with the predictions of the Gaussian Orthogonal Ensemble (GOE) of random matrix theory, whereas quantum analogs of classically integrable systems display the characteristics of the Poisson statistics ${ }^{9)-12)}$. Some results in this direction for field theories have been obtained by Halasz and Verbaarschot: they studied the QCD lattice spectra for staggered fermions and its connection to random matrix theory $^{13)}$.

In this paper we study quantum chaos in a field-theory schematic model. We analyze the energy fluctuation properties of the spatially homogeneous SU(2) Yang-Mills-Higgs (YMH) system (see Ref. 1-4). We show that these fluctuations give a clear quantum signature of the classical chaos-order transition of the system.

The Lagrangian density of the $\mathrm{SU}(2) \mathrm{YMH}$ system $^{14)}$ is given by

$$
L=\frac{1}{2}\left(D_{\mu} \phi\right)^{+}\left(D^{\mu} \phi\right)-V(\phi)-\frac{1}{4} F_{\mu \nu}^{a} F^{\mu \nu a},
$$


where

$$
\begin{gathered}
\left(D_{\mu} \phi\right)=\partial_{\mu} \phi-i g A_{\mu}^{b} T^{b} \phi, \\
F_{\mu \nu}^{a}=\partial_{\mu} A_{\nu}^{a}-\partial_{\nu} A_{\mu}^{a}+g \epsilon^{a b c} A_{\mu}^{b} A_{\nu}^{c},
\end{gathered}
$$

with $T^{b}=\sigma^{b} / 2, b=1,2,3$, generators of the $\mathrm{SU}(2)$ algebra, and where the potential of the scalar field (the Higgs field) is

$$
V(\phi)=\mu^{2}|\phi|^{2}+\lambda|\phi|^{4} .
$$

We work in the (2+1)-dimensional Minkowski space $(\mu=0,1,2)$ and choose spatially homogeneous Yang-Mills and the Higgs fields

$$
\partial_{i} A_{\mu}^{a}=\partial_{i} \phi=0, \quad i=1,2
$$

i.e. we consider the system in the region in which space fluctuations of fields are negligible compared to their time fluctuations.

In the gauge $A_{0}^{a}=0$ and using the real triplet representation for the Higgs field we obtain

$$
\begin{aligned}
L & =\dot{\vec{\phi}}^{2}+\frac{1}{2}\left(\dot{\vec{A}}_{1}^{2}+\dot{\vec{A}}_{2}^{2}\right)-g^{2}\left[\frac{1}{2} \vec{A}_{1}^{2} \vec{A}_{2}^{2}-\frac{1}{2}\left(\overrightarrow{A_{1}} \cdot \vec{A}_{2}\right)^{2}+\right. \\
& \left.+\left(\vec{A}_{1}^{2}+\vec{A}_{2}^{2}\right) \vec{\phi}^{2}-\left(\vec{A}_{1} \cdot \vec{\phi}\right)^{2}-\left(\overrightarrow{A_{2}} \cdot \vec{\phi}\right)^{2}\right]-V(\vec{\phi}),
\end{aligned}
$$

where $\vec{\phi}=\left(\phi^{1}, \phi^{2}, \phi^{3}\right), \vec{A}_{1}=\left(A_{1}^{1}, A_{1}^{2}, A_{1}^{3}\right)$ and $\vec{A}_{2}=\left(A_{2}^{1}, A_{2}^{2}, A_{2}^{3}\right)$.

When $\mu^{2}>0$ the potential $V$ has a minimum at $|\vec{\phi}|=0$, but for $\mu^{2}<0$ the minimum is at

$$
\left|\vec{\phi}_{0}\right|=\sqrt{\frac{-\mu^{2}}{4 \lambda}}=v,
$$

which is the non zero Higgs vacuum. This vacuum is degenerate and after spontaneous symmetry breaking the physical vacuum can be chosen $\vec{\phi}_{0}=$ 
$(0,0, v)$. If $A_{1}^{1}=q_{1}, A_{2}^{2}=q_{2}$ and the other components of the Yang-Mills fields are zero, in the Higgs vacuum the Hamiltonian of the system reads

$$
H=\frac{1}{2}\left(p_{1}^{2}+p_{2}^{2}\right)+g^{2} v^{2}\left(q_{1}^{2}+q_{2}^{2}\right)+\frac{1}{2} g^{2} q_{1}^{2} q_{2}^{2},
$$

where $p_{1}=\dot{q}_{1}$ and $p_{2}=\dot{q}_{2}$. Here $w^{2}=2 g^{2} v^{2}$ is the mass term of the Yang-Mills fields. This YMH Hamiltonian is a toy model for classical non-linear dynamics, with the attractive feature that the model emerges from particle physics. In the next sections we analyze first the classical chaosorder transition of the YMH system and then its connection to the quantal fluctuations of the energy levels.

\section{Classical chaos-order transition}

A classical chaos-order transition for the YMH system has been observed previously by different authors: Savvidy used the Chirikov'criterion ${ }^{1)}$, Kawabe and Ohta studied the Lyapunov exponents ${ }^{3)}$ and Salasnich analyzed the quantal overlapping resonances ${ }^{4)}$. In this paper we study the chaotic behaviour of this YMH system by using the Gaussian curvature criterion of the potential energy ${ }^{16)}$ and the Poincarè Sections ${ }^{17)}$.

At low energy the motion near the minimum of the potential

$$
V\left(q_{1}, q_{2}\right)=g^{2} v^{2}\left(q_{1}^{2}+q_{2}^{2}\right)+\frac{1}{2} g^{2} q_{1}^{2} q_{2}^{2},
$$

where the Gaussian curvature is positive, is periodic or quasiperiodic and is separated from the instability region by a line of zero curvature; if the energy is increased, the system will be for some initial conditions in a region of 
negative curvature, where the motion is chaotic. According to this scenario, the energy $E_{c}$ of chaos-order transition is equal to the minimum value of the line of zero gaussian curvature $K\left(q_{1}, q_{2}\right)$ on the potential-energy surface. For our potential the gaussian curvature vanishes at the points that satisfy the equation

$$
\frac{\partial^{2} V}{\partial q_{1}^{2}} \frac{\partial^{2} V}{\partial q_{2}^{2}}-\left(\frac{\partial^{2} V}{\partial q_{1} \partial q_{2}}\right)^{2}=\left(2 g^{2} v^{2}+g^{2} q_{2}^{2}\right)\left(2 g^{2} v^{2}+g^{2} q_{1}^{2}\right)-4 g^{4} q_{1}^{2} q_{2}^{2}=0
$$

It is easy to show that the minimal energy on the zero-curvature line is given by:

$$
E_{c}=V_{\min }\left(K=0, \overline{q_{1}}\right)=6 g^{2} v^{4}
$$

and by inverting this equation we obtain $v_{c}=\left(E / 6 g^{2}\right)^{1 / 4}$. We conclude that there is a order-chaos transition by increasing the energy $E$ of the system and a chaos-order transition by increasing the value $v$ of the Higgs field in the vacuum (see also Ref. 2). Thus, there is only one transition regulated by the unique parameter $E /\left(g^{2} v^{4}\right)$.

It is important to point out that in general the curvature criterion guarantees only a local instability ${ }^{16)}$ and should therefore be combined with the Poincarè sections ${ }^{17)}$ (see Ref. 18). The classical equations of motion of the YMH system are

$$
\dot{q}_{1}=p_{1}, \quad \dot{q}_{2}=p_{2}, \quad \dot{p}_{1}=-2 g^{2} v^{2} q_{1}-g^{2} q_{1} q_{2}^{2}, \quad \dot{p}_{2}=-2 g^{2} v^{2} q_{2}-g^{2} q_{1}^{2} q_{2}
$$

We use a fourth-order Runge-Kutta method ${ }^{19)}$ to compute the classical trajectories. The conservation of energy restricts any trajectory of the fourdimensional phase space to a three-dimensional energy shell. At a particular 
energy the restriction $q_{1}=0$ defines a two-dimensional surface in the phase space, which is called Poincarè section. Each time a particular trajectory passes through the surface a point is plotted at the position of intersection $\left(q_{2}, p_{2}\right)$. We employ a first-order interpolation process to reduce the inaccuracies due to the use of a finite step length ${ }^{17)}$.

In Figure 1 we plot the Poincarè sections for different values of the Higgs vacuum $v$ but with the same energy $E$ and interaction $g$. Chaotic regions on the surface of section are characterized by a set of randomly distributed points and regular regions by dotted or solid curves. The pictures show that the parameter $v$ plays an important role: for large values it makes the system regular. In fact, if we increase the harmonic part of the YMH potential the effect of the nonlinear term becomes less important. These numerical calculations confirm the analytical predictions of the curvature criterion: with $E=10$ and $g=1$ we get the critical value of the onset of chaos $v_{c}=\left(E / 6 g^{2}\right)^{1 / 4} \simeq 1.14$, in very good agreement with the Poincarè sections.

\section{Quantum signature of the chaos-order tran- sition}

In quantum mechanics the generalized coordinates of the YMH system satisfy the usual commutation rules $\left[\hat{q}_{k}, \hat{p}_{l}\right]=i \delta_{k l}$, with $k, l=1,2$. Introducing the creation and destruction operators

$$
\hat{a}_{k}=\sqrt{\frac{\omega}{2}} \hat{q}_{k}+i \sqrt{\frac{1}{2 \omega}} \hat{p}_{k}, \quad \hat{a}_{k}^{+}=\sqrt{\frac{\omega}{2}} \hat{q}_{k}-i \sqrt{\frac{1}{2 \omega}} \hat{p}_{k},
$$


the quantum YMH Hamiltonian can be written ${ }^{15)}$

$$
\hat{H}=\hat{H}_{0}+\frac{1}{2} g^{2} \hat{V}
$$

where

$$
\begin{gathered}
\hat{H}_{0}=\omega\left(\hat{a}_{1}^{+} \hat{a}_{1}+\hat{a}_{2}^{+} \hat{a}_{2}+1\right), \\
\hat{V}=\frac{1}{4 \omega^{2}}\left(\hat{a}_{1}+\hat{a}_{1}^{+}\right)^{2}\left(\hat{a}_{2}+\hat{a}_{2}^{+}\right)^{2},
\end{gathered}
$$

with $\omega^{2}=2 g^{2} v^{2}$ and $\left[\hat{a}_{k}, \hat{a}_{l}^{+}\right]=\delta_{k l}, k, l=1,2$.

The most used quantity to study the local fluctuations of the energy levels is the spectral statistics $P(s) . P(s)$ is the distribution of nearestneighbour spacings $s_{i}=\left(\tilde{E}_{i+1}-\tilde{E}_{i}\right)$ of the unfolded levels $\tilde{E}_{i}$. It is obtained by accumulating the number of spacings that lie within the bin $(s, s+\Delta s)$ and then normalizing $P(s)$ to unity $^{9)-12)}$.

For quantum systems whose classical analogs are integrable, $P(s)$ is expected to follow the Poisson limit, i.e. $P(s)=\exp (-s)$. On the other hand, quantal analogs of chaotic systems exhibit the spectral properties of GOE with $P(s)=(\pi / 2) s \exp \left(-\frac{\pi}{4} s^{2}\right)$, which is the so-called Wigner distribution ${ }^{9)-12)}$. The distribution $P(s)$ is the best spectral statistics to analyze shorter series of energy levels and the intermediate regions between order and chaos.

Seligman, Verbaarschot and Zirnbauer ${ }^{20)}$ analyzed a class of two-dimensional anharmonic oscillators with polynomial perturbation by using the Brody distribution ${ }^{21)}$

$$
P(s, \omega)=\alpha(\omega+1) s^{\omega} \exp \left(-\alpha s^{\omega+1}\right),
$$


with

$$
\alpha=\left(\Gamma\left[\frac{\omega+2}{\omega+1}\right]\right)^{\omega+1} .
$$

This distribution interpolates between the Poisson distribution $(\omega=0)$ of integrable systems and the Wigner distribution $(\omega=1)$ of chaotic ones, and thus the parameter $\omega$ can be used as a simple quantitative measure of the degree of chaoticity.

We compute the energy levels $\left\{E_{i}\right\}$ with a numerical diagonalization of the truncated matrix of the quantum YMH Hamiltonian in the basis of the harmonic oscillators ${ }^{22)}$. If $\mid n_{1} n_{2}>$ is the basis of the occupation numbers of the two harmonic oscillators, the matrix elements are

$$
<n_{1}^{\prime} n_{2}^{\prime}\left|\hat{H}_{0}\right| n_{1} n_{2}>=\omega\left(n_{1}+n_{2}+1\right) \delta_{n_{1}^{\prime} n_{1}} \delta_{n_{2}^{\prime} n_{2}},
$$

and

$$
\begin{aligned}
< & n_{1}^{\prime} n_{2}^{\prime}|\hat{V}| n_{1} n_{2}>=\frac{1}{4 \omega^{2}}\left[\sqrt{n_{1}\left(n_{1}-1\right)} \delta_{n_{1}^{\prime} n_{1}-2}+\sqrt{\left(n_{1}+1\right)\left(n_{1}+2\right)} \delta_{n_{1}^{\prime} n_{1}+2}+\left(2 n_{1}+1\right) \delta_{n_{1}^{\prime} n_{1}}\right] \times \\
& \times\left[\sqrt{n_{2}\left(n_{2}-1\right)} \delta_{n_{2}^{\prime} n_{2}-2}+\sqrt{\left(n_{2}+1\right)\left(n_{2}+2\right)} \delta_{n_{2}^{\prime} n_{2}+2}+\left(2 n_{2}+1\right) \delta_{n_{2}^{\prime} n_{2}}\right] .
\end{aligned}
$$

The symmetry of the potential enables us to split the Hamiltonian matrix into 4 sub-matrices reducing the computer storage required. These submatrices are related to the parity of the two occupation numbers $n_{1}$ and $n_{2}$ : even-even, odd--odd, even-odd, odd--even. The numerical energy levels depend on the dimension of the truncated matrix: we compute the numerical levels in double precision increasing the matrix dimension until the first 100 levels converge within 8 digits (matrix dimension $1156 \times 1156)^{22), 23)}$.

We use the first 100 energy levels of the 4 sub-matrices to calculate the $P(s)$ distribution. In order to remove the secular variation of the level density 
as a function of the energy $E$, for each value of the coupling constant the corresponding spectrum is mapped, by a numerical procedure described in Ref. 24 , into one which has a constant level density: $\left\{E_{i}\right\} \rightarrow\left\{\tilde{E}_{i}\right\}$ (unfolding procedure). We use the following standard procedure to avoid mixing between states of different symmetry classes: 1) the diagonalization is performed for each sub-matrix (first 100 levels for each sub-matrix); 2) the unfolding is done for each sub-matrix; 3 ) the spacings are calculated for each sub-matrix; 4) the spacings of the 4 sub-matrices are accumulated to plot the $\mathrm{P}(\mathrm{s})$ distribution.

In Figure 2 we plot the $P(s)$ distribution for different values of the parameter $v$. The figure shows a Wigner-Poisson transition by increasing the value $v$ of the Higgs field in the vacuum. By using the $\mathrm{P}(\mathrm{s})$ distribution and the Brody function it is possible to give a quantitative measure of the degree of quantal chaoticity of the system. Our numerical calculations show clearly the quantum chaos-order transition and its connection to the classical one.

\section{Conclusions}

The chaotic behaviour of an homogenous YMH system has been studied both in classical and quantum mechanics. The Gaussian curvature criterion and the Poincarè sections show that the chaotic behaviour is regulated by the unique parameter $E /\left(g^{2} v^{4}\right)$. The YMH system has a order-chaos transition by increasing the energy $E$ and a chaos-order transition by increasing the value $v$ of the Higgs field in the vacuum.

The nearest-neighbour spacing distribution of the energy levels confirms with great accuracy the classical chaos-order transition of the YMH system. 
In particular, the Brody function shows a Wigner-Poisson transition for the $P(s)$ distribution in correspondence to the classical chaos-order transition.

We observe that, as stressed previously, our YMH system is a toy model but it is very useful because it is possible to compare classical to quantum chaos. In the future will be important to study classical and quantum chaos in more realistic field theories.

\section{Acknowledgments}

The author is grateful to G. Benettin, V.R. Manfredi, M. Robnik and A. Vicini for stimulating discussions. 


\section{Figure Captions}

Figure 1: The Poincarè sections of the model. From the top: $v=1, v=1.1$ and $v=1.2$. Energy $E=10$ and interaction $g=1$.

Figure 2: $P(s)$ distribution. From the top: $v=1(\omega=0.92), v=1.1$ $(\omega=0.34)$ and $v=1.2(\omega=0.01)$, where $\omega$ is the Brody parameter. First 100 energy levels and interaction $g=1$. The dotted, dashed and solid curves stand for Wigner, Poisson and Brody distributions, respectively. 


\section{References}

1. G.K. Savvidy, Nucl. Phys. B 246, 302 (1984).

2. A. Gorski, Acta Phys. Pol. B 15, 465 (1984).

3. T. Kawabe and S. Ohta, Phys. Rev. D 44, 1274 (1991).

4. L. Salasnich, Phys. Rev. D 52, 6189 (1995).

5. T. Kawabe, Phys. Lett. B 343, 254 (1995).

6. L. Salasnich, Mod. Phys. Lett. A 10, 3119 (1995).

7. J. Segar and M.S. Sriram, Phys. Rev. D 53, 3976 (1996).

8. S.G. Matinyan and B. Muller, Phys. Rev. Lett. 78, 2515 (1997).

9. M.C. Gutzwiller, Chaos in Classical and Quantum Mechanics (Springer, Berlin, 1990).

10. A.M. Ozorio de Almeida, Hamiltonian Systems: Chaos and Quantization (Cambridge University Press, Cambridge, 1990).

11. K. Nakamura, Quantum Chaos (Cambridge Nonlinear Science Series, Cambridge, 1993).

12. G. Casati and B.V. Chirikov, Quantum Chaos (Cambridge University Press, Cambridge, 1995).

13. M.A. Halasz and J.J.M. Verbaarschot, Phys. Rev. Lett. 74, 3920 (1995). 
14. C. Itzykson and J.B. Zuber, Quantum Field Theory (McGraw-Hill, New York, 1985).

15. G.K. Savvidy, Phys. Lett. B 159, 325 (1985).

16. M. Toda, Phys. Lett. A 48, 335 (1974).

17. M. Henon, Physica D 5, 412 (1982).

18. G. Benettin, R. Brambilla and L. Galgani, Physica A 87, 381 (1977).

19. Subroutine D02BAF, The NAG Fortran Library, Mark 14 (NAG Ltd, Oxford, 1990).

20. T.H. Seligman, J.J.M. Verbaarschot and M.R. Zirnbauer, Phys. Rev. lett. 53, 215 (1984).

21. T.A. Brody, Lett. Nuovo Cimento 7, 482 (1973).

22. S. Graffi, V.R. Manfredi and L. Salasnich, Mod. Phys. Lett. B 9, 747 (1995).

23. Subroutine F02AAF, The NAG Fortran Library, Mark 14 (NAG Ltd, Oxford, 1990).

24. V.R. Manfredi, Lett. Nuovo Cimento 40, 135 (1984). 